\title{
Impact of implementing a fast-track protocol and standardized guideline for the management of pediatric appendicitis
}

\author{
Jennifer Y. Lam, MD \\ Paul Beaudry, MD, MSc \\ Brett A. Simms, PhD \\ Mary E. Brindle, MD, MPH
}

Accepted July 6, 2020

\author{
Correspondence to: \\ J. Lam \\ Division of Pediatric Surgery \\ Western University \\ Children's Hospital at London \\ Health Sciences Centre \\ 800 Commissioners Rd E \\ London ON N6A 5W9 \\ jennifery.lam@Ihsc.on.ca
}

DOI: $10.1503 /$ cjs.005420

\begin{abstract}
Background: In 2017, a provincial guideline was created to fast track and standardize care for pediatric appendicitis in Alberta. We conducted a study to determine the impact of implementation of the guideline at our institution on length of stay (LOS), antibiotic stewardship efforts and costs.

Methods: We performed a retrospective review of the charts of all patients younger than 18 years of age who underwent appendectomy at our institution in 2 periods: before guideline implementation (Dec. 1, 2016, to May 31, 2017) and after implementation (Dec. 1, 2017, to May 31, 2018). We compared LOS, duration of antibiotic therapy, 30-day postdischarge complication rates and variable cost between the 2 cohorts.

Results: Of the 276 total appendectomy procedures performed, 185 were for simple appendicitis (81 before guideline implementation and 104 after implementation), and 91 were for complicated appendicitis (44 and 47, respectively). The median LOS was shorter in the postimplementation cohort for both simple and complicated appendicitis (15.5 h [interquartile range (IQR) 12-19 h] v. $17.0 \mathrm{~h}[$ [QR $13-22 \mathrm{~h}], p=0.03$; and $3.0 \mathrm{~d}$ [IQR $2-4 \mathrm{~d}$ ] v. $3.0 \mathrm{~d}$ [IQR 3-5 d], $p=0.05$, respectively). Patients with complicated appendicitis had fewer antibiotic days after guideline implementation; the difference was statistically significant for patients without diffuse peritoneal contamination or abscess formation $(p=0.02)$. There were no differences between the cohorts with respect to 30-day rates of complications, including emergency department visits, readmission and surgical site infections. After guideline implementation, the average variable cost per patient was reduced by $\$ 230$, equating to a total average annual cost savings of $\$ 75842$ for our institution.
\end{abstract}

Conclusion: The implementation of a provincial guideline aimed at standardizing care in pediatric appendicitis at our institution was associated with shortened LOS, improved antibiotic stewardship efforts and reduced cost of care. Other institutions may replicate our model of a standardized pathway in the management of pediatric appendicitis in an effort to improve the quality of patient care and reduce health care costs.

Contexte : En 2017, des lignes directrices provinciales ont vu le jour en Alberta afin d'accélérer et de normaliser les soins pédiatriques pour appendicite. Notre étude visait à déterminer l'effet de leur application, par notre établissement, sur la durée du séjour, la gestion des antibiotiques et les coûts des soins.

Méthodes : Nous avons examiné de façon rétrospective le dossier de tous les patients de moins de 18 ans ayant subi une appendicectomie à notre établissement avant l'application des lignes directrices (entre le $1^{\text {er }}$ décembre 2016 et le 31 mai 2017) et après (entre le $1^{\text {er }}$ décembre 2017 et le 31 mai 2018). Les données relatives à la durée du séjour, à la durée de l'antibiothérapie, au taux de complications 30 jours après le congé et aux coûts variables ont été comparées entre les 2 groupes.

Résultats : Des 276 appendicectomies totales effectuées, 185 concernaient une appendicite simple (81 avant l'application des lignes directrices et 104 après), et 91 , une appendicite compliquée (44 avant l'application et 47 après). La durée médiane du séjour était plus courte dans le groupe postapplication, tant pour l'appendicite simple (15,5 h [écart interquartile (EI) 12-19 h] c. $17,0 \mathrm{~h}[\mathrm{EI} 13-22 \mathrm{~h}] ; p=0,03)$ que pour l'appendicite compliquée $(3,0 \mathrm{j}[\mathrm{EI} 2-4 \mathrm{j}] \mathrm{c}$. 3,0 j [EI $3-5 j] ; p=0,05)$. Les patients qui présentaient une appendicite compliquée avaient une antibiothérapie moins longue après l'application des lignes directrices; la différence était statistiquement significative chez les patients sans contamination péritonéale diffuse ou abcès $(p=0,02)$. Aucune différence n'a été observée entre les cohortes en ce qui a trait au taux de complications à 30 jours, qui comprenait les consultations à l'urgence, les réadmissions et les infections du site opératoire. L'application des lignes directrices a permis de réduire les coûts variables par patient de $230 \$$, ce qui représente une économie annuelle moyenne de 75842 \$ pour notre établissement.

Conclusion : L'application des lignes directrices provinciales visant à normaliser les soins pédiatriques pour appendicite a été associée, dans notre établissement, à une réduction de la durée du séjour, à l'amélioration de la gestion des antibiotiques et à une diminution des coûts des soins. D'autres établissements pourraient reproduire ce modèle de soins normalisés pour améliorer la qualité et réduire les coûts. 
A cute appendicitis is the most common pediatric abdominal emergency. ${ }^{1-5}$ The lifetime risk of appendicitis is $7 \%-8 \%$, with the highest incidence between the ages of 10 and 19 years. ${ }^{3,4}$ Appendicitis can be categorized as simple/nonperforated or complicated, with perforation or abscess formation or both. The management of appendicitis depends on presentation but also varies by treating surgeon. Same-day discharge for pediatric simple appendicitis is safe; however, the uptake of this practice is inconsistent. ${ }^{1,4,6-9}$ Treatment of complicated appendicitis is even more variable, with inconsistent durations of antibiotic courses and hospital stays. ${ }^{10}$

To promote the delivery of patient care practices based on evidence-informed provincial standards, in 2015, Alberta Health Services (the provincial health authority) introduced a province-wide Clinical Knowledge \& Content Management program. ${ }^{11}$ The program partnered with Strategic Clinical Networks and provincial content experts to create best-practice, evidence-informed guidelines. ${ }^{11}$ In April 2017, our team led the creation of the provincial pediatric appendicitis guideline under the Clinical Knowledge \& Content Management umbrella. ${ }^{5}$

We used an iterative process to incorporate knowledge and feedback from the existing literature, pediatric and adult general surgeons representing all major community and academic hospitals in the province, emergency medicine, nursing leads and clinical support services (e.g., laboratory, diagnostic imaging). The guideline incorporates evidence to support the assessment and management of pediatric appendicitis, and provides order sets to streamline care and recommendations on antibiotic duration. It also provides the rationale for and steps to implement a fast-track protocol for simple appendicitis, including standardization of a nursing-led discharge process (i.e., written orders to allow nurses to discharge patients based on specific clinical criteria provided concurrently with other postoperative orders) and education points for patients and families. ${ }^{5}$ After multidisciplinary stakeholder approval by the pediatric general surgery team and nursing leads of the day surgery unit and surgical ward, the guideline was implemented at the Alberta Children's Hospital, Calgary, in November 2017. Importantly, the guideline was created with knowledge of resources and infrastructure within Alberta, and the Alberta Children's Hospital implementation strategy took institutional factors into consideration.

We conducted a study to determine the impact of implementation of the guideline at our institution on length of stay (LOS), antibiotic stewardship efforts and costs. We hypothesized that a standardized approach to appendicitis management within a formal provincial guideline coupled with a strategy of rollout and ongoing operationalization supported by principles of implementation science would streamline care, improve health care provider buy-in for fast-track protocols, and, ulti- mately, improve overall outcomes and reduce cost in the management of pediatric appendicitis.

\section{Methods}

After receiving institutional ethics approval, we retrospectively compared two 6-month periods during which pediatric patients underwent appendectomy at Alberta Children's Hospital: before implementation of the appendicitis guideline (Dec. 1, 2016, to May 31, 2017) and after implementation (Dec. 1, 2017, to May 31, 2018). The intervening 6-month period, which coincided with the time frame of guideline creation, was excluded as a washout period, as there may have been partial early adoption of optimal practices by the team.

Children (age $<18 \mathrm{yr}$ ) treated for appendicitis with appendectomy at Alberta Children's Hospital were included. Those who underwent incidental appendectomy (i.e., part of a Ladd procedure or other intestinal resection) or whose appendectomy or majority of treatment occurred at a different centre were excluded. Simple versus complicated appendicitis was determined by the surgeon at the time of surgery and identified through cross-referencing operative notes and postoperative orders. We reviewed all patient charts to collect demographic characteristics and outcome measures.

The primary outcome for patients with simple appendicitis was postappendectomy length of stay (LOS), specifically, the number of hours from surgery to discharge and the proportion discharged within 12 hours. The primary outcome for patients with complicated appendicitis was LOS. Secondary outcomes for all patients included appropriate prophylactic antibiotic administration (both timing and proper regimen), rates of 30-day postdischarge complications, including calls to the surgery clinic, emergency department visits, readmission, reoperation, surgical site infections, Clostridium difficile infections, ileus, bowel obstructions, and postoperative abscess or phlegmon formation, as well as variable costs.

One of us (J.Y.L.) reviewed the electronic medical record (EMR) of all patients, cross-referencing results of laboratory work, microbiology studies and diagnostic imaging tests with readmission diagnoses and treatment, to identify any complications within the 30 -day postdischarge period. The EMR provides both inpatient and outpatient records, including care obtained from urgent care centres in the city. We calculated variable costs per patient by summing costs of all ordered items (laboratory and diagnostic imaging tests, consultations, medications) identified from the EMR, beginning from presentation in the emergency department to discharge, as well as physician claims and operating room supplies. Variable costs do not include fixed costs such as cost of a bed, nursing salary, food and utilities. An additional secondary outcome for patients with complicated appendicitis was total antibiotic days, including intravenous and oral formulations. 
All patients with presumed simple appendicitis or their parent/guardian were counselled preoperatively about the potential of early discharge if criteria were met. If the discharge criteria were not being met, or if there were patient or family concerns or social considerations, the fast-track protocol was not implemented.

To assess for surgeon adoption of the fast-track protocol, we evaluated the EMR of all patients with simple appendicitis in the postimplementation group to determine whether the standardized nursing-led discharge orders were written concurrently with postoperative orders. Regardless of discharge timing, all patients were included in the analysis.

\section{Statistical analysis}

We analyzed the data as 2 separate subsets: simple and complicated appendicitis. We performed a further subgroup analysis of outcomes for patients with complicated appendicitis by classifying patients into 2 groups - perforated or gangrenous versus diffuse peritoneal contamination or abscess - based on different treatment pathways for differing disease severity suggested by the provincial guideline. Data for continuous variables are presented as means and standard deviation, or medians and interquartile range (IQR). We used a 2-tailed Student $t$ test for means and the Mann-Whitney $U$ test for nonparametric data, and compared categoric variables using the $\chi^{2}$ or Fisher exact test. We analyzed the data analyses using SPSS software, version 25 (IBM Corporation). Statistical significance was defined as a 2 -tailed $p \leq 0.05$.

\section{Results}

A total of 276 appendectomy procedures were performed over the course of the 2 study periods, 185 for simple appendicitis ( 81 before guideline implementation and 104 after implementation) and 91 for complicated appendicitis (44 and 47, respectively). Patient demographic characteristics, including age and sex, were similar between the preand postimplementation cohorts (Table 1). There was a trend toward increased administration of appropriate prophylactic antibiotics in the postimplementation group; however, this did not reach statistical significance $(68.8 \%$ v. $78.1 \%, p=0.08)$. All patients underwent laparoscopic appendectomy with the exception of a patient with complicated appendicitis in the preimplementation cohort, who underwent open appendectomy. The patient's young age $(2 \mathrm{yr})$, intraperitoneal sepsis and ileus necessitated an open appendectomy procedure as a result of lack of intraabdominal domain.

\section{Simple appendicitis}

The median LOS was significantly shorter in the postimplementation group than in the preimplementation group (15.5 h [IQR 12-19 h] v. $17.0 \mathrm{~h}$ [IQR 13-22 h], $p=0.03)$, and the proportion discharged within 12 hours was significantly greater in the postimplementation group than in the preimplementation group $(37.5 \%$ v. $21.0 \%$, $p=0.02)$. Length of stay and proportion discharged within 12 hours were similar for all units and wards in the preimplementation cohort (Table 2). However, after

\begin{tabular}{|c|c|c|c|c|}
\hline \multirow[b]{2}{*}{ Characteristic } & \multicolumn{3}{|c|}{ No. $(\%)$ of patients* } & \multirow[b]{2}{*}{$p$ value } \\
\hline & $\begin{array}{c}\text { Total } \\
n=276\end{array}$ & $\begin{array}{l}\text { Before implementation } \\
\qquad n=125\end{array}$ & $\begin{array}{l}\text { After implementation } \\
\qquad n=151\end{array}$ & \\
\hline Age, mean $\pm S D, y r$ & $11 \pm 3.6$ & $10.8 \pm 3.5$ & $11.1 \pm 3.8$ & 0.4 \\
\hline Male sex & $170(61.6)$ & $75(60.0)$ & $95(62.9)$ & 0.6 \\
\hline Appropriate antibiotic prophylaxis & $204(73.9)$ & 86 (68.8) & $118(78.1)$ & 0.08 \\
\hline \multicolumn{5}{|l|}{ Indication } \\
\hline Simple appendicitis & $185(67.0)$ & $81(64.8)$ & $104(68.9)$ & - \\
\hline Complicated appendicitis & $91(33.0)$ & $44(35.2)$ & $47(31.1)$ & - \\
\hline
\end{tabular}

Table 2. Length of stay for patients with simple appendicitis before and after guideline implementation, by treating unit

\begin{tabular}{llcccccc} 
& \multicolumn{3}{c}{ Before implementation } & & \multicolumn{3}{c}{ After implementation } \\
\cline { 2 - 3 } Outcome & $\begin{array}{c}\text { Day surgery } \\
n=43\end{array}$ & $\begin{array}{c}\text { Ward } \\
n=38\end{array}$ & $p$ value & $\begin{array}{c}\text { Day surgery } \\
n=60\end{array}$ & $\begin{array}{c}\text { Ward } \\
n=44\end{array}$ & $p$ value \\
\hline Length of stay, median (IQR), $\mathrm{h}$ & $14(13-22)$ & $18(16-22)$ & 0.4 & & $14(10-17)$ & $18(12-22)$ & 0.003 \\
\hline Discharge within $12 \mathrm{~h}, \mathrm{no} .(\%)$ of patients & $9(21)$ & $8(21)$ & 1.0 & $27(45)$ & $12(27)$ & 0.07 \\
\hline IQR = interquartile range. & & & & & & \\
\hline
\end{tabular}




\begin{tabular}{|c|c|c|c|c|c|c|}
\hline \multirow[b]{3}{*}{ Complication } & \multicolumn{6}{|c|}{ No. (\%) of patients } \\
\hline & \multicolumn{3}{|c|}{ Simple appendicitis } & \multicolumn{3}{|c|}{ Complicated appendicitis } \\
\hline & $\begin{array}{c}\text { Before } \\
\text { implementation } \\
n=81\end{array}$ & $\begin{array}{c}\text { After } \\
\text { implementation } \\
n=104\end{array}$ & $p$ value & $\begin{array}{c}\text { Before } \\
\text { implementation } \\
n=44\end{array}$ & $\begin{array}{c}\text { After } \\
\text { implementation } \\
n=47\end{array}$ & $p$ value \\
\hline Call to surgery clinic & $6(7.4)$ & 4 (3.8) & 0.3 & $0(0.0)$ & $2(4.3)$ & 0.5 \\
\hline Emergency department visit & $13(16.0)$ & $16(15.4)$ & 0.9 & $7(15.9)$ & $7(14.9)$ & 1.0 \\
\hline Unplanned diagnostic imaging test & $5(6.2)$ & $9(8.6)$ & 0.6 & $5(11.4)$ & $8(17.0)$ & 0.6 \\
\hline Readmission & $2(2.5)$ & $6(5.8)$ & 0.5 & $2(4.5)$ & $5(10.6)$ & 0.4 \\
\hline Reintervention & $0(0.0)$ & $1(1.0)$ & 1.0 & $0(0.0)$ & $1(2.1)$ & 1.0 \\
\hline Wound infection & $3(3.7)$ & $3(2.9)$ & 1.0 & $3(6.8)$ & $0(0.0)$ & 0.1 \\
\hline Clostridium difficile infection & $0(0.0)$ & $0(0.0)$ & & $1(2.3)$ & $0(0.0)$ & 0.5 \\
\hline Intra-abdominal abscess/phlegmon & $0(0.0)$ & $0(0.0)$ & & $2(4.5)$ & $3(6.4)$ & 1.0 \\
\hline lleus & $1(1.2)$ & $1(1.0)$ & 1.0 & $1(2.3)$ & $0(0.0)$ & 0.5 \\
\hline Small bowel obstruction & $0(0.0)$ & $0(0.0)$ & - & $0(0.0)$ & $1(2.1)$ & 1.0 \\
\hline
\end{tabular}

implementation, a significantly higher proportion of patients admitted to the day surgery unit than to any other ward were discharged within 12 hours $(45.0 \%$ v. $27.3 \%$, $p=0.07)$, and day surgery patients had a significantly shorter LOS (14.0 h [IQR 10-17 h] v. 18.0 h [IQR $12-22 \mathrm{~h}], p=0.003)$.

In the postimplementation cohort, 96 patients $(92.3 \%)$ received the standardized nursing-led discharge orders as part of their postoperative orders. Of the 65 patients not discharged within 12 hours, 19 (29.2\%) were found to have specific clinical or social concerns responsible for the delay in discharge.

There were no statistically significant differences in 30-day postdischarge complication rates between the preand postimplementation groups (Table 3). In particular, the rates of emergency department visits were $16.0 \%$ and $15.4 \%$, respectively $(p=0.9)$, and the readmission rates were $2.5 \%$ and $5.8 \%$, respectively $(p=0.5)$.

\section{Complicated appendicitis}

The median LOS in the postimplementation cohort was 3.0 (IQR 2-4) days, compared to 3.0 (IQR 3-5) days in the preimplementation cohort $(p=0.05)$ (Table 4$)$. Patients in the postimplementation cohort had a median of 6.5 (IQR 2-10) total antibiotic days, compared to 8.0 (IQR 7-10) days in the preimplementation cohort $(p=0.07)$. Patients with perforated or gangrenous appendicitis had a significantly shorter median LOS $(2.0 \mathrm{~d}$ [IQR 1-3 d] v. $3.0 \mathrm{~d}$ [IQR 2-4 d], $p=0.04)$ and median number of antibiotic days (2.0 [IQR 1-7] v. 7.0 [IQR $2-10], p=0.02$ ) after guideline implementation than before implementation (Table 4). There were no differences between the pre- and postimplementation cohorts in LOS or antibiotic days in the diffuse peritoneal contamination or abscess subgroup. There were no statistically significant differences in 30-day postdischarge complication rates between the pre- and postimplementa-
Table 4. Length of stay and duration of antibiotic therapy among patients with complicated appendicitis before and after guideline implementation, overall and by severity of disease

\begin{tabular}{|c|c|c|c|}
\hline \multirow[b]{2}{*}{ Outcome } & \multicolumn{2}{|c|}{ Median (IQR)* } & \multirow[b]{2}{*}{$p$ value } \\
\hline & $\begin{array}{c}\text { Before } \\
\text { implementation }\end{array}$ & $\begin{array}{c}\text { After } \\
\text { implementation }\end{array}$ & \\
\hline \multicolumn{4}{|l|}{ Overall } \\
\hline No. of patients & 44 & 47 & \\
\hline Length of stay, $d$ & $3.0(3-5)$ & $3.0(2-4)$ & 0.05 \\
\hline $\begin{array}{l}\text { Duration of antibiotic } \\
\text { therapy, } d\end{array}$ & $8.0(7-10)$ & $6.5(2-10)$ & 0.07 \\
\hline \multicolumn{4}{|l|}{ Gangrenous/perforated } \\
\hline No. of patients & 26 & 26 & \\
\hline Length of stay, $d$ & $3.0(2-4)$ & $2.0(1-3)$ & 0.04 \\
\hline $\begin{array}{l}\text { Duration of antibiotic } \\
\text { therapy, } d\end{array}$ & $7.0(2-10)$ & $2.0(1-7)$ & 0.02 \\
\hline \multicolumn{4}{|l|}{$\begin{array}{l}\text { Diffuse peritoneal } \\
\text { contamination/abscess }\end{array}$} \\
\hline No. of patients & 18 & 21 & \\
\hline Length of stay, $d$ & $4.5(3-10)$ & $3.0(3-5.5)$ & 0.1 \\
\hline $\begin{array}{l}\text { Duration of antibiotic } \\
\text { therapy, } d\end{array}$ & $10.0(7-12)$ & $10.0(7-12)$ & 0.5 \\
\hline $\begin{array}{l}\text { IQR = interquartile range. } \\
{ }^{*} \text { Except where noted otherw }\end{array}$ & & & \\
\hline
\end{tabular}

tion cohorts (Table 3). Specifically, the rates of emergency department visits were $15.9 \%$ and $14.9 \%$, respectively $(p=1.0)$, and the readmission rates were $4.5 \%$ and $10.6 \%$, respectively $(p=0.4)$.

\section{Variable costs}

The average variable cost was $\$ 3667$ before guideline implementation and \$3437 after implementation, for an average savings of $\$ 230$ per patient. The average variable cost was $\$ 110$ lower per patient for simple appendicitis and \$516 lower per patient for complicated appendicitis. Based on the average annual appendicitis rates from preceding years, the total yearly cost savings 
from implementing the appendicitis pathway at Alberta Children's Hospital was $\$ 75842$.

\section{Discussion}

This study adds to the growing literature supporting the safe and effective use of fast-track protocols for pediatric simple appendicitis. . $^{1,4-6}$ The use of a formal provincial pediatric appendicitis guideline was effective at reducing LOS in our population. The proportion of patients with simple appendicitis discharged within 12 hours after appendectomy almost doubled, from $21.0 \%$ to $37.5 \%$, without an increase in rates of 30-day complications, including emergency department visits.

We believe the structured approach to guideline creation and use of sound implementation science principles, including developing a quality culture, having multidisciplinary stakeholder participation, and providing resources and education to front-line health care providers, improved buy-in of the fast-track protocol. This is evidenced not only by the increase in patients discharged within 12 hours, but also the high rate $(92 \%)$ of surgeon adoption of the standardized nursing-led discharge orders. Before implementation of the appendicitis guideline, individual practitioners could provide conditional discharge orders for the nursing team to carry out. However, the lack of structure in orders, standardization of discharge criteria and health care provider education on fast-track discharge protocols did not allow for the optimal application of early-discharge practices.

We found a stark contrast in nursing uptake of the fast-track protocol between the day surgery unit and other hospital wards. This finding is most likely explained by the usual workflow of staff within the respective units. The daily work of our day surgery nurses revolves around preparing, caring for and discharging patients undergoing elective surgery. They are accustomed to the outpatient nature of surgery and are familiar with quick turnaround rates. This aligns perfectly with the approach required of fast-track protocols. Conversely, our ward nurses are accustomed to sick inpatients, who tend to stay longer in hospital; therefore, the nurses may not be attuned to the quick turnaround associated with fasttrack protocols. This shows the value of established short-stay or day surgery units in the successful execution of fast-track protocols, while recognizing that further education may enhance familiarity and successful practice of fast-track protocols on other units. The provincial appendicitis guideline should help with ongoing efforts to educate health care providers, as well as provide information and resources to reinforce the practice of fast-track protocols.

Although a rate of discharge within 12 hours of $37.5 \%$ may seem relatively low, it is within the documented range of fast-track and same-day discharge adoption rates, $28 \%-$
$80 \% .4,6-8$ In addition, the average rate of outpatient management of appendicitis across National Surgical Quality Improvement Program Pediatric participating centres in 2015 was $48 \% .^{1}$ The wide range of adoption rates can be explained by the inconsistent definition of same-day discharge. Traditionally, same-day discharge encompasses only patients discharged within the same calendar day, but others define it as discharge within 24 hours after a procedure. $^{1,6-9}$ To avoid this ambiguity, at our institution, we use the fast-track nomenclature and clearly define compliance as discharge within 12 hours. We feel this time frame is reasonable to consider as a fast-track discharge, as it captures patients who are discharged early in the morning after appendectomy the night before and thus would not qualify under the traditional definition. It is important to include these patients, as early-morning discharge still increases bed capacity for the daily census and can act as an efficiency indicator.

Another important consideration is the substantial distance that many patients must travel to pediatric centres like ours to receive treatment. It may be inappropriate to discharge these patients early under a fast-track protocol, given the distance they would have to travel to return if necessary. With this in mind, it is crucial to select the appropriate patients and families for fast-track protocols.

In the present study, overall, patients with complicated appendicitis had a shorter LOS and a trend toward reduced total antibiotic days after implementation of the provincial appendicitis guideline. In the subgroup analysis, patients with perforated or gangrenous appendicitis had both a significantly shorter LOS and significantly fewer total antibiotic days. These findings were not surprising, as it was noted during guideline creation that substantial variation in care practices existed among surgeons, particularly regarding management of the gangrenous or minimally contaminated perforated appendix. It is evident that practice variation can lead to inconsistent care, including potentially unnecessary inpatient days and longer antibiotic courses. This study shows that following standardized guidelines for complicated appendicitis can streamline care to improve outcomes and antibiotic stewardship efforts, particularly in patients with gangrenous or minimally contaminated perforated appendicitis. It also appears that reducing the LOS for appropriate patients did not lead to increased rates of emergency department visits or readmission.

There was an increase in appropriate use (both timing and appropriate regimen) of prophylactic antibiotics, from $69 \%$ to $78 \%$, after guideline implementation. In most cases in which the patient missed the prophylactic dose, the reason was timing: patients who received treatment antibiotics on admission were not redosed within the 60-minute window for surgical site infection prophylaxis. Further education regarding the ideal regimen and 
timing of antibiotic administration should help improve rates of surgical site infection prophylaxis.

There was no difference in 30-day complication rates between the pre- and postimplementation cohorts, which suggests that shorter LOS and antibiotic courses did not negatively affect outcomes. However, both cohorts had a surprisingly high rate (about 15\%) of emergency department visits within 30 days after discharge. A much smaller proportion required readmission or therapy (reintervention or medical treatment, including analgesia and antibiotic therapy). Improved communication of discharge instructions and follow-up options may help to decrease the burden placed on the emergency department by transitioning some visits into telephone calls or ambulatory clinic visits. $^{12}$

We identified an overall reduction in average variable costs associated with managing pediatric appendicitis after guideline implementation. The advantage of using variable costs over total costs is that activity-based costing provides a measure of only those aspects of care over which physicians have control and thus could be influenced by a standardized pathway. Predictably, cost savings were greater among patients with complicated appendicitis, as there is typically more variation and inconsistent care in this population. Furthermore, our results likely underestimate the true cost savings, as fixed costs such as utilities and nursing salary are not represented in our costing algorithm and would contribute to the cost differential between cohorts, considering the overall shorter LOS in the postimplementation cohort. These results are important as we proceed with efforts targeting the triple aim: improving population health, enhancing patient experience and reducing costs. ${ }^{13}$

Future aims include improving rates of appropriate surgical site infection prophylaxis, further training for ward nursing teams to improve fast-track protocol adoption, and more comprehensive discharge instructions to decrease use of emergency department resources.

\section{Limitations}

The retrospective and single-institution nature and relatively small sample are limitations of this study. More patients may be needed to detect differences in complication rates. However, we feel that our findings are generalizable to other pediatric centres, specifically, that use of formal appendicitis guidelines for both simple and complicated appendicitis standardizes patient care and may improve outcomes. In addition, although our outcome measures included 30-day emergency department visits and readmission, our comparison of costs purely reflected those of the initial hospital admission. Given similar rates before and after guideline implementation, we do not expect that this would have affected our estimate substantially.

\section{Conclusion}

Implementation of fast-track protocols in simple appendicitis and standardized care pathways for complicated appendicitis can lead to shortened LOS, improved antibiotic stewardship and reduced cost. Success in adopting similar pathways may be optimized by an approach similar to that undertaken province-wide in Alberta, based on principles of implementation science including the use of institutional mandates and leadership support, a multidisciplinary approach to developing formal system-wide guidelines, and the use of educational resources and order sets for front-line health care providers.

Affiliations: From the Division of Pediatric Surgery, University of Calgary, Alberta Children's Hospital, Calgary, Alta. (Lam, Beaudry, Brindle); and Clinical Analytics, Alberta Health Services, Alberta Children's Hospital, Calgary, Alta. (Simms).

Competing interests: None declared.

Contributors: Jennifer Lam and Mary Brindle designed the study. Jennifer Lam and Brett Simms acquired the data, which Jennifer Lam, Paul Beaudry and Mary Brindle analyzed. Jennifer Lam wrote the manuscript, which all authors critically revised. All authors gave final approval of the article to be published.

Content licence: This is an Open Access article distributed in accordance with the terms of the Creative Commons Attribution (CC BYNC-ND 4.0) licence, which permits use, distribution and reproduction in any medium, provided that the original publication is properly cited, the use is noncommercial (i.e., research or educational use), and no modifications or adaptations are made. See: https://creativecommons. org/licenses/by-nc-nd/4.0/.

\section{References}

1. Litz CN, Stone L, Alessi R, et al. Impact of outpatient management following appendectomy for acute appendicitis: an ACS NSQIP-P analysis. 7 Pediatr Surg 2018;53:625-8.

2. Bos C, Doumouras AG, Akhtar-Danesh GG, et al. A populationbased cohort examining factors affecting all-cause morbidity and cost after pediatric appendectomy: Does annual adult procedure volume matter? Am 7 Surg 2019;218:619-23.

3. Pepper VK, Stanfill AB, Pearl RH. Diagnosis and management of pediatric appendicitis, intussusception, and Meckel diverticulum. Surg Clin North Am 2012;92:505-26.

4. Halter JM, Mallory B, Neilson IR, et al. Same-day discharge following laparoscopic appendectomy for uncomplicated acute appendicitis as a measure of quality in the pediatric population. 7 Laparoendosc Adv Surg Tech A 2016;26:309-13.

5. Alberta Health Services. Provincial clinical knowledge topic: appendicitis, pediatric - inpatient. V 1.0. Edmonton: Alberta Health Services; 2018. Available: https://extranet.ahsnet.ca/teams/policydocuments/1/ klink/et-klink-ckv-appendicitis-pediatric-inpatient.pdf (accessed 2019 Nov. 26).

6. Aguayo P, Alemayehu H, Desai AA, et al. Initial experience with same day discharge after laparoscopic appendectomy for nonperforated appendicitis. 7 Surg Res 2014;190:93-7.

7. Grewal H, Sweat J, Vazquez WD. Laparoscopic appendectomy in children can be done as a fast-track or same-day surgery. FSLS 2004; 8:151-4.

8. Alkhoury F, Malvezzi L, Knight CG, et al. Routine same-day discharge after acute or interval appendectomy in children: a prospective study. Arch Surg 2012;147:443-6. 
9. Oyetunji TA, Gonzalez DO, Aguayo P, et al. Variability in sameday discharge for pediatric appendicitis. 7 Surg Res 2015;199: 159-63.

10. Lee SL, Islam S, Cassidy LD, et al.; 2010 American Pediatric Surgical Association Outcomes and Clinical Trials Committee. Antibiotics and appendicitis in the pediatric population: an American Pediatric Surgical Association Outcomes and Clinical Trials Committee Systematic Review. 7 Peds Surg 2010;45: 2181-5.

11. Alberta Health Services. Clinical Knowledge \& Content Manage- ment (CKCM) Service. Edmonton: Alberta Health Services; 2020. Available: https://www.albertahealthservices.ca/cgv/Page15918.aspx (accessed 2021 May 21).

12. Cameron DB, Serres SK, Glass CC, et al. Leveraging the incidence, burden, and fiscal implications of unplanned hospital revisits for the prioritization of prevention efforts in pediatric surgery. Ann Surg 2020;271:191-9.

13. Berwick DM, Nolan TW, Whittington J. The triple aim: care, health, and cost. Health Aff (Millwood) 2008;27:759-69. 